\title{
Experiences of health care transition voiced by young adults with type I diabetes: a qualitative study
}

This article was published in the following Dove Press journal:

Adolescent Health, Medicine and Therapeutics

20 October 2014

Number of times this article has been viewed

\author{
Katharine C Garvey ${ }^{1,5}$ \\ Margaret G Beste ${ }^{2}$ \\ Donna Luff ${ }^{3,5}$ \\ Astrid Atakov-Castillo 2 \\ Howard A Wolpert ${ }^{2,5}$ \\ Marilyn D Ritholz ${ }^{4-6}$ \\ 'Division of Endocrinology, Boston \\ Children's Hospital, Boston, MA, \\ USA; ${ }^{2}$ Adult Section, Joslin Diabetes \\ Center, Boston, MA, USA; ${ }^{3}$ Institute \\ for Professionalism and Ethical \\ Practice, Boston Children's Hospital, \\ Boston, MA, USA; ${ }^{4}$ Behavioral and \\ Mental Health Unit, Joslin Diabetes \\ Center, Boston, MA, USA; ${ }^{5}$ Harvard \\ Medical School, Boston, MA, USA; \\ ${ }^{6}$ Department of Psychiatry, Boston \\ Children's Hospital, Boston, MA, USA
}

Correspondence: Katharine C Garvey Division of Endocrinology, Boston Children's Hospital, 300 Longwood Avenue, Boston, MA 02115, USA Tel + I 6179193045

Email katharine.garvey@childrens. harvard.edu
Objective: This qualitative study aimed to explore the experience of transition from pediatric to adult diabetes care reported by posttransition emerging adults with type 1 diabetes (T1D), with a focus on preparation for the actual transfer in care.

Methods: Twenty-six T1D emerging adults (mean age $26.2 \pm 2.5$ years) receiving adult diabetes care at a single center participated in five focus groups stratified by two levels of current glycemic control. A multidisciplinary team coded transcripts and conducted thematic analysis.

Results: Four key themes on the process of transfer to adult care emerged from a thematic analysis: 1) nonpurposeful transition (patients reported a lack of transition preparation by pediatric providers for the transfer to adult diabetes care); 2) vulnerability in the college years (patients conveyed periods of loss to follow-up during college and described health risks and diabetes management challenges specific to the college years that were inadequately addressed by pediatric or adult providers); 3) unexpected differences between pediatric and adult health care systems (patients were surprised by the different feel of adult diabetes care, especially with regards to an increased focus on diabetes complications); and 4) patients' wish list for improving the transition process (patients recommended enhanced pediatric transition counseling, implementation of adult clinic orientation programs, and peer support for transitioning patients).

Conclusion: Our findings identify modifiable deficiencies in the T1D transition process and underscore the importance of a planned transition with enhanced preparation by pediatric clinics as well as developmentally tailored patient orientation in the adult clinic setting.

Keywords: young adults, chronic illness, type 1 diabetes mellitus, transition to adult care

\section{Introduction}

According to contemporary developmental theories, young adulthood does not immediately follow adolescence, but starts when individuals are in their late 20 s or early 30s. The developmental stage between ages $\sim 18$ and 30 years defines a period called "emerging adulthood."1 The emerging adulthood period is typified by competing educational, social, occupational, and economic demands and presents special challenges for patients with type 1 diabetes (T1D), a chronic illness that requires frequent medical follow-up and ongoing intensive self-care. ${ }^{1-3}$ As emerging adults with T1D experience competing life priorities and decreasing parental support, adherence to self-care often declines, and glycemic control may deteriorate. Consequently, emerging adults with T1D are at risk for adverse health outcomes, including: acute diabetes complications, such as ketoacidosis and severe hypoglycemia; chronic microvascular complications, such as nephropathy and retinopathy; and early mortality. ${ }^{4-10}$ 
Health care transition has been defined as "the planned, purposeful movement of emerging adults from child-centered to adult-oriented health care systems." "11 Key milestones of this period include transition preparation, engagement in chronic disease self-management, and the actual transfer of health care from pediatric to adult systems. In 2002, a health care transition consensus statement from American Academy of Pediatrics, the American Academy of Family Physicians, and the American College of Physicians emphasized the importance of concerted transition planning by both primary care and subspecialty providers caring for adolescents with chronic illness. ${ }^{12}$

However, in the intervening years, the implementation of transition care has been inadequate; recently updated 2011 guidelines cited a lack of progress and restated the priority of transition coordination as a basic standard of high-quality medical care. ${ }^{13}$

There is a broad consensus that the lack of effective transition from pediatric to adult diabetes care may contribute to the fragmentation of health care and increased risk for adverse outcomes in emerging adults with T1D. ${ }^{3,14}$ Prior research has highlighted difficulties in the T1D transition process, including delays in care, ${ }^{15-19}$ suboptimal transition preparation, ${ }^{16}$ increased posttransition hospitalization, ${ }^{20}$ and patient dissatisfaction with the transition experience. ${ }^{15-19}$ In 2011, the American Diabetes Association published expert consensus guidelines on health care transition for emerging adults with $\mathrm{T} 1 \mathrm{D},{ }^{3}$ but empiric data are limited on transition processes and outcomes, especially in the US. ${ }^{10,16,21-24}$

Qualitative research offers a unique opportunity for patient experiences to inform the design of effective interventions to improve T1D transition care. We previously published qualitative data focusing on emerging adult perceptions of patient-provider relationships across the transition from pediatric to adult T1D care. ${ }^{25}$ The objective of our qualitative analysis in the current study, from the same patient sample, was to analyze experiences of transition preparation and care transfer as voiced by posttransition emerging adults with T1D.

\section{Methods}

\section{Study design}

We conducted a series of five focus groups to gain insight into the emerging adults' shared experience and the understanding of the transition from pediatric to adult diabetes care. Qualitative focus group methodology facilitates the study of multiple experiences, perceptions, and emotions within a group setting. ${ }^{26,27}$ For our study, focus group methodology was selected to obtain detail and context about the emerging adults' lived experience of health care transition that would be enhanced by the group members' dynamic interactions with one another. The institutional committee on human subjects approved this study.

\section{Sample}

We utilized purposive sampling strategies ${ }^{28}$ (ie, selection based on knowledge of a population and objective of the study) to identify emerging adults with T1D, ages 22-30 years old, diagnosed with diabetes at $\leq 18$ years, and now receiving care in the adult diabetes clinic at a comprehensive diabetes center in Boston, MA, USA. We selected this age range to ensure establishment of care in the adult clinic, given the mean age of transition to adult care (19-20 years) in published US T1D cohorts. ${ }^{10,16}$

Although the diabetes center has both pediatric and adult units, there is no structured health care transition program. Boston has a large number of colleges and universities, and the adult diabetes clinic treats many emerging adult patients who moved to the region for education or employment after receiving pediatric diabetes care in other geographic areas.

The study staff contacted eligible patients and invited them to participate in the appropriate focus group. An introductory letter was mailed to all eligible patients and followed by individual phone calls. Groups were formed, based on the patients' level of glycemic control, (hemoglobin $\mathrm{A}_{1 \mathrm{c}}$; Tosoh analyzer; Tosoh Medics Inc., Foster City, CA, USA) as measured at the clinic visit most proximal to recruitment. Subjects were grouped by lower $\mathrm{A}_{1 \mathrm{c}}(\leq 8.5 \%)$ and higher $\mathrm{A}_{1 \mathrm{c}}(>8.5 \%)$. Subjects with similar glycemic control were grouped together to establish group homogeneity, which allows for increased compatibility, cohesion, and disclosure in focus groups ${ }^{29}$ and may serve to lessen discomfort or competition in discussion of disease self-management. All study subjects provided written informed consent and received compensation for their time.

\section{Focus group guide and procedures}

We developed a structured focus group guide based on an extensive review of the academic medical literature and interviews with pediatric and adult diabetes providers. Investigators developing the guide included a pediatric endocrinologist, adult endocrinologist, and a clinical psychologist who had extensive clinical experience with transitioning emerging adults. The focus group guide applied broad, openended questions to elicit details of the transition experience from patient perspective. Further, the guide was iteratively 
revised after the first focus group in light of emerging topics expressed within the group. Key focus group guide items included the following: reasons for transition; experiences leaving the pediatric diabetes team and receiving care in the adult clinic; definitions of transition success; current diabetes support system and parental role; degree of independence in diabetes self-care; current barriers to diabetes control; and the impact of the health care transition on the perceptions of diabetes.

Each focus group was moderated by the same investigator (MDR), who ensured that each group fully discussed each topic in the guide and that all subjects had the opportunity to voice their perspective. A second investigator (KCG) was the comoderator and wrote field notes to capture key points and observations about the group discussion and interactions. Focus group discussions lasted 75 minutes. At the end of each group, the moderator and comoderator met to share observations. Each session was audiotaped and transcribed verbatim by three team members within 4-8 weeks of each session. To validate the transcriptions, the focus group comoderator performed a quality check of the transcribed files while listening to the recordings.

\section{Data analysis}

Over the course of 1 year, the multidisciplinary analysis team, consisting of a clinical psychologist, pediatric endocrinologist, nutritionist/diabetes researcher, and medical sociologist, met at regular intervals to analyze data according to the principles of thematic analysis. ${ }^{30-32}$ Team members had previously conducted qualitative research studies, and two team members (MDR and DL), who were well-versed in qualitative research methods, guided the analytic process.

At the start of data analysis, each team member independently read all of the transcripts and open-coded the data by marking and categorizing key words and phrases to generate initial codes. Initial codes were discussed by the group, and discrepancies were resolved through consensus to develop the preliminary thematic framework. Initial coding and revision through consensus is a common early stage in inductive thematic analysis. Each team member then applied this thematic framework to all transcripts. After transcripts were coded and reviewed, one member of the research team entered the coded transcripts into NVivo 8 software (QSR International Pty Ltd, Victoria, Australia), and codes were then further grouped and organized. The team reviewed the organized themes and concluded that theoretical saturation was reached; no new themes were generated from the later focus groups. Finally, the team met to agree on the definitive themes. An audit trail tracked the decision-making process and supported the dependability of the data.

\section{Results \\ Participant characteristics}

Five focus groups were conducted, each consisting of three to seven subjects, $(n=26)$. The mean age of subjects was $26.2 \pm 2.5$ years, and diabetes duration was $16.3 \pm 4.7$ years. Age at transition from pediatric to adult diabetes care was $20.3 \pm 3.2$ years. The majority of the subjects was female (62\%), college-educated (85\%), and Caucasian (81\%). Three groups had lower $\mathrm{A}_{1 \mathrm{c}}$ values (mean for lower $\mathrm{A}_{1 \mathrm{c}}$ groups $=7.4 \% \pm 0.6 \%$ ), and two groups had higher $A_{1 c}$ values (mean $=9.8 \% \pm 1.0 \%$ ).

Nine subjects $(35 \%)$ reported receiving their pediatric diabetes care at the pediatric unit of the same diabetes center where they presently received adult care. Of the remaining 17 participants, eight received their diabetes care from other hospitals in the same city, one from a pediatrician in the same state, and eight from pediatric providers out of state. None of the participants had participated in any structured health care transition programs.

\section{Thematic analysis}

Four key themes on the health care transition process emerged from thematic analysis: 1) nonpurposeful transition; 2) vulnerability in the college years; 3) unexpected differences between pediatric and adult care systems; and 4) patients' wish list for improving the transition process. During the process of thematic analysis, the study team reached a consensus that none of these themes differed by level of current glycemic control. In the following sections, illustrative quotations are provided for each theme; subject age and sex are included with each quotation.

\section{Nonpurposeful transition}

Patients in all groups perceived an absence of purposeful preparation for the transition to adult diabetes care. Many patients reported having to find a new adult provider on their own. Few participants experienced any formal coordination of care between pediatric and adult diabetes providers:

He's [the pediatric diabetes doctor] telling you he can't see you anymore, you're too old; you're going to have to find somebody else. [male, age 24]

I didn't really have any sort of hand-holding or anything. [female, age 23]

I did not receive any recommendation. I think the thought process was, once you transition out, however the new 
organization you find manages the transition [...] they'll

just take care of it there. [male, age 27]

While most subjects did not receive specific adult care referrals, those who did receive adult clinic recommendations nonetheless desired more structure and support in scheduling the first appointment and choosing a provider. Even those patients who felt eager for change still described needing more help with the actual process of adult care establishment:

The woman I spoke with when I called the [adult] clinic booked me an appointment with whoever had openings and that person automatically became my provider. I didn't feel like I had any choice in it. [female, age 24]

There really wasn't much discussion about it. Other than where I was going to go. Yeah, I just kind of had to take it from there. [female, age 25]

\section{Vulnerability in the college years}

Patients described periods of interrupted or decreased clinical follow-up during their college education years. Despite college being acknowledged by patients as a period characterized by increased independence, separation from family, and challenges with diabetes self-care, many agreed that they "got away with" the minimum frequency of diabetes visits:

During college, I went to the endocrinologist once a year, and I didn't really do much with the information, and nobody really noticed. [female, age 27]

I felt like I came [to clinic] through college just to get my $A_{1 c}[\ldots]$ he [provider] didn't know what my life was like, and $[\ldots]$ you guys know that college and diabetes is not the easiest time. [female, age 27]

Further exploring this issue, patients shared a concern that typical diabetes visit content did not feel relevant to the diabetes self-care difficulties experienced during college. Specifically, patients felt that health risk behaviors, such as sexuality and alcohol use, as well as dietary excursions, were inadequately addressed by diabetes providers:

All throughout college, the biggest thing I struggled with is balancing my health care and social life. [female, age 23]

Moving to college [...] there are issues of, you know, drinking, and what happens when you eat half a pizza at $3 \mathrm{am}$, and when you're stressed with finals. These are the things that are not discussed openly and honestly [with providers] [female, age 25]
Of note, the desire for more developmentally appropriate care during the college years was expressed both by patients who transferred to adult care before or during college, as well as those who transferred after college:

There was this age gap, like between age 18 and 22, when there were very specific lifestyle things that never got discussed either in pediatrics or adult. [female, age 25]

It's [college] a vulnerable time [...] whoever your doctor is, just because you're assigned as an adult doesn't mean you're necessarily ready to face adult situations [...]. Your doctor needs to say, 'Hey, you're an adult, here are some adult-type situations you're going to run into.' [male, age 27]

\section{Unexpected differences between pediatric and adult health care systems}

Patients frequently chose words such as "shock" or "surprise" when referring to their initial impressions of the adult diabetes clinic setting. A common thread in these discussions was the absence of expectations set by pediatric providers prior to transfer:

I didn't really understand that it would be different $[\ldots]$

I didn't have a lot of warning [...] that is what may have been so shocking at that first visit. [female, age 23]

The most shocking thing was coming in the first time, and it wasn't the standard procedure I was used to. Yeah, it's just something you have to kind of pick up [...]. It was just very different. [female, age 25]

Patients felt unprepared for the focus on an older patient population inherent to a standard adult clinical program. Many commented on the higher prevalence of type 2 diabetes patients in adult care:

I remember sort of feeling [...] that I was one of the only type $1 \mathrm{~s}$ in the room, and it was all of a sudden very much a fish out of water experience [...] it felt like much more of the focus was on type 2. [female, age 25]

In addition, patients felt overwhelmed by the increased exposure to chronic diabetes complications in the new adult clinic setting:

That is a huge shock, when you come in and you see these elderly people with walkers and canes and everything, and you're like [...] OK [..] is that going to be me in 6 years? Like, am I going to still be here? And it was kind of overwhelming to go from one end of the spectrum to the opposite. [female, age 27] 
The complications [...] you don't see them as much when you're seeing your childhood doctor [...] I remember the first time I came here, I hate to say it, but you see a lot more of them [complications] - it's scary. This is serious. [female, age 25]

\section{Patient wish list for improving the transition process}

Focus group participants energetically collaborated to propose recommendations for transition process improvement. Patients recommended more robust transition counseling with expectation-setting by pediatric providers regarding the culture of adult care. In addition, patients stressed the importance of targeted referrals to specific adult diabetes providers and adult providers with expertise in emerging adult care:

If I could make a recommendation to [pediatric providers] regarding pediatric care it would be, even if they only have one adult provider that they recommend, have somebody that you recommend [...] I wish that I had received that from my pediatric care $[\ldots]$ just something as simple as that would have made a world of difference. [male, age 27]

I think what would be a huge benefit would be to have specific providers who target that age group and have the young adult postcollege mindset. [female, age 23]

In addition, patients recommended programming to orient emerging adults to both the logistics and the care practices of the new adult clinic settings, to help shape expectations and support adjustment:

At least, you know, having somebody say ' $\mathrm{OK}$, this is [...] where things are, where the lab is. This is where the waiting room is.' Instead of that first appointment where you're meeting your doctor and on top of that trying to find your way around. [female, age 27]

I would have liked some form of warning, like a packet of information or something. [female, age 23]

How about one of the nurses, like every Monday night call all the incoming young adults? Or I would be willing to call like a 17-year-old or whatever and be like 'this is what it's like.' [female, age 27]

Finally, patients felt that peer mentoring from other emerging adults who had navigated the health care transition would be an invaluable source of support:

I'm just a lone person going through diabetes, seeing a new diabetes doctor, and feeling like I didn't have that network. [female, age 29]
I would have liked to have had some kind of support system when I entered into adult care. I remember asking my doctor, 'are there any other college students that are doing this as well?' [female, age 24]

\section{Discussion}

This study details the experiences of transfer to adult care as voiced by emerging adults with $\mathrm{T} 1 \mathrm{D}$ receiving adult care at a specialty diabetes center. The interactive communication between focus group members facilitated the emergence of several clear themes concerning the challenges in the process of transfer from pediatric to adult care. In our qualitative analysis, the key themes all related to the need for more systematic, purposeful, developmentally tailored transition preparation, including explicit education on high-risk behaviors for the college-aged group and open dialogue about the cultural differences between pediatric and adult care systems.

Regarding our first theme of nonpurposeful preparation for transfer by pediatric providers, our study adds patient voices to support results from previous survey-based research in the US and abroad demonstrating: suboptimal transition preparation; ${ }^{16}$ difficulties with the establishment of adult diabetes care ${ }^{24,33}$ and patient dissatisfaction with the transition experience. ${ }^{15-19}$ Our sample was a relatively advantaged group of patients who have found their way to a specialized adult diabetes center from a variety of different pediatric centers. Despite the fact that they have established adult diabetes care, these patients did not credit their successful establishment of adult diabetes care to specific pediatric preparation. Rather, most patients expressed dissatisfaction with perceived passivity or lack of hand-holding from pediatric teams regarding transfer coordination and felt that they were left to their own devices to establish adult care.

Interestingly, in our previously published analyses on the patient-provider relationship across transition, a major theme of loss and gain in provider relationships differed by the level of glycemic control; patients with lower $\mathrm{A}_{1 \mathrm{c}}$ levels felt more reluctance to leave their pediatric providers, while those with higher $A_{1 c}$ wanted to transfer to adult care. ${ }^{25}$ Nonetheless, in the current analysis, the pervasive theme of nonpurposeful transition, or lack of well-coordinated transfer planning, did not differ among groups with higher or lower current $A_{1 c}$ levels. We suspect that the lack of formal transition planning would be further magnified in other emerging adult populations from more diverse socioeconomic backgrounds; an important area for future study. 
Patients with limited transition preparation have been shown to be at higher risk for gaps between pediatric and adult care, ${ }^{33}$ and previous research has shown an increased incidence of poor glycemic control and acute and chronic diabetes complications in youth with infrequent clinic follow-up. ${ }^{7,34}$ Importantly, because a lack of purposeful transition preparation may lead to fragmentation of health care and increased risk for adverse health outcomes, the development of programs to systematize transition preparation is a critical area for future intervention research.

We also found that patients identified an important vulnerability in diabetes transition care during the college years, a new finding that warrants further exploration. Patients' feelings that they "got away with" reduced medical visits and that "nobody noticed" require further exploration and underscore the importance of health care providers (both pediatric and adult) understanding and addressing this phenomenon. In the US, where there is no broadly mandated transition age, our findings support the need to assess the readiness of each individual patient for adult services prior to transition, and the idea that delaying transfer until during or after college might improve visit frequency and glycemic control outcomes.

For example, a recent 1 year prospective study of high school seniors found that patients who stayed in pediatric diabetes care (compared to those who transitioned to adult care or were already in adult care) demonstrated the best diabetes self-care and did not experience declines in glycemic control over time. ${ }^{22}$

In our analysis, whether patients were describing experiences in pediatric or adult care, the focus of diabetes medical visits in the college years frequently did not feel relevant to their lives, and high-risk lifestyle behaviors were not adequately uncovered or addressed. The diabetes literature is limited in this area, but it does point to a number of patientreported barriers to self-care during college. ${ }^{35,36}$ While these issues are important to address during medical visits in any emerging adult population, specific increased health risks are present in the setting of diabetes (eg, the risk of complications from poor glycemic control in pregnancy or the risk of hypoglycemic seizure with alcohol abuse). Moreover, as research in developmental psychology demonstrates, emerging adults often pursue high-risk experiences more freely than adolescents because they are less likely to be monitored by parents and can pursue them more freely than adults because they are less constrained by roles. ${ }^{1}$

In T1D populations, emerging adults who classify themselves as adults have been shown to have more adaptive coping skills and a lower $\mathrm{A}_{1 \mathrm{c}}$ level compared to those endorsing nonadult classification. ${ }^{37}$ Our results highlight the need for specific provider education and programs to address the lifestyle needs and high-risk behaviors of college students with T1D.

We previously demonstrated that the posttransition emerging adults with T1D in this sample perceived improved partnership and shared decision-making with adult diabetes providers. ${ }^{25}$ However, despite these positive patient-provider relational attributes, in the current analysis we uncovered a pervasive theme of shock regarding the differences between pediatric and adult services. Notably, rather than focusing on the individual patient-provider relationship, these perceived differences and the accompanying surprise were rooted in systems-based factors, such as an increased presence of type 2 diabetes or diabetes complications in adult services. Our findings may help guide the clinical content of pretransition diabetes visits as well as shape the practice of adult providers accepting transitioning T1D patients. Qualitative work in the UK and Australia has highlighted very similar differences in emerging adults' perceptions of pediatric versus adult diabetes care. ${ }^{38,39}$ Along similar lines, research in health care transition in general pediatrics and complex care populations has suggested that pediatric and adult care systems represent two different medical subcultures, and that the emerging adults' lack of preparation for successful participation in the adult health care system may contribute to problems with transition. ${ }^{40}$

In our analysis, the patients' wish list for transition process improvement centered on new recommendations to demystify the adult care terrain; in addition to enhanced pediatric transition counseling, patients recommended programs to orient emerging adults to adult diabetes clinic, as well as peer support programs for emerging adults in adult care.

There are several limitations to this study. Recall bias is a concern. While the mean transition age of participants was 20.3 years, a number of years had nonetheless elapsed since transition for many participants; as a result, despite the importance of understanding posttransition perceptions, the recall of specific details is subject to potential omission or bias. Further, we did not conduct respondent validation of the findings, which may have generated additional data and further insights into the credibility of the final themes. Because our study voices the experiences of a relatively advantaged sample of patients receiving diabetes care at a specialized center, future research should apply these themes to other subsets of emerging adults and purposively select different samples in terms of sex, age, ethnicity, and care location. Future research should consider providers' and parents' perspectives on transition. However, the logic of qualitative 
sampling rests not on generalizability or representativeness, but on the notion of transferability, or whether the results advance theoretical understandings and are relevant to multiple situations. ${ }^{41}$ A qualitative study design is not ideally suited to compare transition experiences in patients with differing levels of glycemic control. Future work should employ quantitative methods to prospectively assess glycemic control, clinic attendance, self-care adherence, patient satisfaction, and socioeconomic variables across transition.

In conclusion, our results may help guide the development, implementation, and refinement of interventions to improve the process of transition from pediatric to adult diabetes care for emerging adults with T1D. Previous work has demonstrated the promise of dedicated emerging adult diabetes clinics with pediatric and adult providers working together. ${ }^{24,42-44}$ However, in settings where such a dedicated transition clinic is not feasible, lower-cost interventions to promote purposeful transition counseling, education about college and high-risk lifestyle behaviors, and acclimation to the adult care landscape should be implemented and tested in both pediatric and adult diabetes care settings.

\section{Acknowledgments}

Dr Garvey was supported by: the Agency for Healthcare Research and Quality (T32-HS000063); the National Institute of Diabetes and Digestive and Kidney Diseases (T32DK007699-30); the National Institute for Diabetes and Digestive and Kidney Diseases (K12-DK094721); and the William Randolph Hearst Foundation.

\section{Disclosure}

The authors report no conflicts of interest in this work.

\section{References}

1. Arnett JJ. Emerging adulthood. A theory of development from the late teens through the twenties. Am Psychol. 2000;55(5):469-480.

2. American Diabetes Association. Standards of medical care in diabetes 2013. Diabetes Care. 2013;36 Suppl 1:S11-S66.

3. Peters A, Laffel L; American Diabetes Association Transitions Working Group. Diabetes care for emerging adults: recommendations for transition from pediatric to adult diabetes care systems: a position statement of the American Diabetes Association, with representation by the American College of Osteopathic Family Physicians, the American Academy of Pediatrics, the American Association of Clinical Endocrinologists, the American Osteopathic Association, the Centers for Disease Control and Prevention, Children with Diabetes, The Endocrine Society, the International Society for Pediatric and Adolescent Diabetes, Juvenile Diabetes Research Foundation International, the National Diabetes Education Program, and the Pediatric Endocrine Society (formerly Lawson Wilkins Pediatric Endocrine Society). Diabetes Care. 2011;34(11):2477-2485.

4. Bryden KS, Dunger DB, Mayou RA, Peveler RC, Neil HA. Poor prognosis of young adults with type 1 diabetes: a longitudinal study. Diabetes Care. 2003;26(4):1052-1057.
5. Bryden KS, Peveler RC, Stein A, Neil A, Mayou RA, Dunger DB, Clinical and psychological course of diabetes from adolescence to young adulthood: a longitudinal cohort study. Diabetes Care. 2001;24(9):1536-1540.

6. Laing SP, Swerdlow AJ, Slater SD, et al. The British Diabetic Association Cohort Study, I: all-cause mortality in patients with insulintreated diabetes mellitus. Diabet Med. 1999;16(6):459-465.

7. Jacobson AM, Hauser ST, Willett J, Wolfsdorf JI, Herman L. Consequences of irregular versus continuous medical follow-up in children and adolescents with insulin-dependent diabetes mellitus. J Pediatr. 1997;131(5):727-733.

8. Wills CJ, Scott A, Swift PG, Davies MJ, Mackie AD, Mansell P. Retrospective review of care and outcomes in young adults with type 1 diabetes. BMJ. 2003;327(7409):260-261.

9. Laing SP, Jones ME, Swerdlow AJ, Burden AC, Gatling W. Psychosocial and socioeconomic risk factors for premature death in young people with type 1 diabetes. Diabetes Care. 2005;28(7):1618-1623.

10. Lotstein DS, Seid M, Klingensmith G, et al; SEARCH for Diabetes in Youth Study Group. Transition from pediatric to adult care for youth diagnosed with type 1 diabetes in adolescence. Pediatrics. 2013;131(4): e1062-e1070.

11. Blum RW, Garell D, Hodgman CH, et al. Transition from child-centered to adult health-care systems for adolescents with chronic conditions. A position paper of the Society for Adolescent Medicine. J Adolesc Health. 1993;14(7):570-576.

12. American Academy of Pediatrics; American Academy of Family Physicians; American College of Physicians-American Society of Internal Medicine. A consensus statement on health care transitions for young adults with special health care needs. Pediatrics. 2002; 110(6 Pt 2):1304-1306.

13. Cooley WC, Sagerman PJ; American Academy of Pediatrics; American Academy of Family Physicians; American College of Physicians; Transitions Clinical Report Authoring Group. Supporting the health care transition from adolescence to adulthood in the medical home. Pediatrics. 2011;128(1):182-200.

14. Weissberg-Benchell J, Wolpert H, Anderson BJ. Transitioning from pediatric to adult care: a new approach to the post-adolescent young person with type 1 diabetes. Diabetes Care. 2007;30(10):2441-2446.

15. Busse FP, Hiermann P, Galler A, et al. Evaluation of patients' opinion and metabolic control after transfer of young adults with type 1 diabetes from a pediatric diabetes clinic to adult care. Horm Res. 2007;67(3):132-138.

16. Garvey KC, Wolpert HA, Rhodes ET, et al. Health care transition in patients with type 1 diabetes: young adult experiences and relationship to glycemic control. Diabetes Care. 2012;35(8):1716-1722.

17. Kipps S, Bahu T, Ong K, et al. Current methods of transfer of young people with Type 1 diabetes to adult services. Diabet Med. 2002; 19(8):649-654.

18. Pacaud D, McConnell B, Huot C, Aebi C, Yale J. Transition from pediatric care to adult care for insulin-dependent diabetes patients. Can $J$ Diabetes. 1996;20(4):14-20.

19. Pacaud D, Yale JF, Stephure D, Trussell R, Davies HD. Problems in transition from pediatric to adult care in individuals with diabetes. Can J Diabetes. 2005;29(1):13-18.

20. Nakhla M, Daneman D, To T, Paradis G, Guttmann A. Transition to adult care for youths with diabetes mellitus: findings from a Universal Health Care System. Pediatrics. 2009;124(6):e1134-e1141.

21. Hanna KM, Weaver MT, Stump TE, et al. Initial findings: primary diabetes care responsibility among emerging adults with type 1 diabetes post high school and move out of parental home. Child Care Health Dev. 2013;39(1):61-68.

22. Helgeson VS, Reynolds KA, Snyder PR, et al. Characterizing the transition from paediatric to adult care among emerging adults with Type 1 diabetes. Diabet Med. 2013;30(5):610-615.

23. Insabella G, Grey M, Knafl G, Tamborlane W. The transition to young adulthood in youth with type 1 diabetes on intensive treatment. Pediatr Diabetes. 2007;8(4):228-234. 
24. Hilliard ME, Perlus JG, Clark LM, et al. Perspectives from before and after the pediatric to adult care transition: a mixed-methods study in type 1 diabetes. Diabetes Care. 2014;37(2):346-354.

25. Ritholz MD, Wolpert H, Beste M, Atakov-Castillo A, Luff D, Garvey KC. Patient-provider relationships across the transition from pediatric to adult diabetes care: a qualitative study. Diabetes Educ. 2014;40(1):40-47.

26. Morgan D. Focus Groups as Qualitative Research. Newbury Park, CA: Sage Publications Inc.; 1988.

27. Krueger RA, Casey MA. Focus Groups: A Practical Guide for Applied Research. 3rd ed. Thousand Oaks, CA: Sage Publications Inc.; 2000.

28. Rice PL, Ezzy D. Qualitative Research Methods: A Health Focus. Oxford: Oxford University Press; 1999.

29. Fern EF. Advanced Focus Group Research. Thousand Oaks, CA: Sage Publications Inc.; 2001.

30. Mays N, Pope C. Qualitative research in health care. Assessing quality in qualitative research. BMJ. 2000;320(7226):50-52.

31. Boyatzis RE. Transforming Qualitative Information: Thematic Analysis and Code Development. Thousand Oaks, CA: Sage Publications Inc.; 1998.

32. Braun V, Clarke V. Using thematic analysis in psychology. Qual Res Psychol. 2006;3(2):77-101.

33. Garvey KC, Wolpert HA, Laffel LM, Rhodes ET, Wolfsdorf JI, Finkelstein JA. Health care transition in young adults with type 1 diabetes: barriers to timely establishment of adult diabetes care. Endocr Pract. 2013;19(6):946-952.

34. Holmes-Walker DJ, Llewellyn AC, Farrell K. A transition care programme which improves diabetes control and reduces hospital admission rates in young adults with Type 1 diabetes aged 15-25 years. Diabet Med. 2007;24(7):764-769.
35. Wdowik MJ, Kendall PA, Harris MA. College students with diabetes: using focus groups and interviews to determine psychosocial issues and barriers to control. Diabetes Educ. 1997;23(5):558-562.

36. Wdowik MJ, Kendall PA, Harris MA, Auld G. Expanded health belief model predicts diabetes self-management in college students. J Nutr Educ. 2001;33(1):17-23.

37. Luyckx K, Moons P, Weets I. Self-classification as an adult in patients with type 1 diabetes: relationships with glycemic control and illness coping. Patient Educ Couns. 2011;85(2):245-250.

38. Dovey-Pearce G, Hurrell R, May C, Walker C, Doherty Y. Young adults' (16-25 years) suggestions for providing developmentally appropriate diabetes services: a qualitative study. Health Soc Care Community. 2005;13(5):409-419.

39. Visentin K, Koch T, Kralik D. Adolescents with Type 1 Diabetes: transition between diabetes services. J Clin Nurs. 2006;15(6):761-769.

40. Reiss JG, Gibson RW, Walker LR. Health care transition: youth, family, and provider perspectives. Pediatrics. 2005;115(1):112-120.

41. Kuper A, Reeves S, Levinson W. An introduction to reading and appraising qualitative research. BMJ. 2008;337:a288.

42. Lane JT, Ferguson A, Hall J, et al. Glycemic control over 3 years in a young adult clinic for patients with type 1 diabetes. Diabetes Res Clin Pract. 2007;78(3):385-391.

43. Logan J, Peralta E, Brown K, Moffett M, Advani A, Leech N. Smoothing the transition from paediatric to adult services in type 1 diabetes. J Diabetes Nurs. 2008;12(9):328-338.

44. Orr DP, Fineberg NS, Gray DL. Glycemic control and transfer of health care among adolescents with insulin dependent diabetes mellitus. J Adolesc Health . 1996;18(1):44-47.
Adolescent Health, Medicine and Therapeutics

\section{Publish your work in this journal}

Adolescent Health, Medicine and Therapeutics is an international, peer-reviewed, open access journal focusing on health, pathology, and treatment issues specific to the adolescent age group. All aspects of health maintenance, preventative measures and disease treatment interventions are addressed within the journal and practitioners from

\section{Dovepress}

all disciplines are invited to submit their work as well as healthcare researchers and patient support groups.. The manuscript management system is completely online and includes a very quick and fair peerreview system. Visit http://www.dovepress.com/testimonials.php to read real quotes from published authors. 